\title{
eJRIEPS
}

Ejournal de la recherche sur l'intervention en éducation physique et sport

Hors-série $N^{\circ} 2 \mid 2018$

À propos de certaines bases théoriques et pratiques des sports collectifs

\section{Vers une littératie motrice en sport collectif}

Jean-Francis Gréhaigne

\section{OpenEdition}

Journals

Édition électronique

URL : http://journals.openedition.org/ejrieps/505

DOI : $10.4000 /$ ejrieps. 505

ISSN : 2105-0821

Éditeur

ELLIADD

Référence électronique

Jean-Francis Gréhaigne, « Vers une littératie motrice en sport collectif », eJRIEPS [En ligne], Hors-série $N^{\circ} 2$ | 2018, mis en ligne le 01 juillet 2018, consulté le 01 août 2019. URL : http:// journals.openedition.org/ejrieps/505; DOI : 10.4000/ejrieps.505

La revue eJRIEPS est mise à disposition selon les termes de la Creative Commons Attribution 4.0 International License. 


\section{Vers une littératie motrice en sport collectif}

Le problème des connaissances et des compétences motrices que doivent construire les élèves et les étudiants en vue d'une vie physique d'adulte équilibrée reste un problème essentiel voire vital pour l'éducation physique et sportive (EPS). En Amérique du Nord, une dizaine d'années en arrière, le terme «Physical Literacy » (savoir-faire physique voire alphabétisation physique) était assez peu utilisé. En effet, on parlait plutôt d'exercice, de condition physique ou d'activité physique ou de loisir pour représenter un mode de vie actif. Aujourd'hui, le constat est tout autre : la littératie physique s'est répandue largement chez les coachs, les professionnels du loisir et les chercheurs du domaine des activités physiques. Chez les anglophones (Almond, 2013 ; Mandigo \& Holt, 2004), la littératie physique renvoie à la motivation, la confiance, la compétence physique, le savoir et la compréhension qu'une personne possède et qui lui permettent de valoriser et de prendre en charge son engagement envers sa pratique d'activité physique pour toute sa vie.

En France, l'accent a été mis sur des pratiques scolaires adaptées et diversifiées d'activités physiques, sportives et artistiques qui doivent concourir à l'épanouissement des élèves. Les enseignements proposés par l'éducation physique et sportive participent au maintien ou développement de la condition physique et à la bonne santé tout au long de la vie tout en développant des valeurs citoyennes. Ils permettent un engagement corporel qui représente, pour beaucoup de jeunes, la seule pratique d'une activité physique et vise à savoir gérer sa vie physique d'adulte (Programme lycée, 2010 Bulletin officiel spécial nº 4 du 29 avril 2010). L'EPS permet à l'élève d'assurer sa sécurité et celle des autres, d'entretenir sa santé, de développer l'image et l'estime de soi pour construire sa relation aux autres. Elle concourt à la recherche du bien-être, de la santé et de la forme physique et doit amener l'élève à bâtir une image positive de son corps. Grace au plaisir éprouvé, aux efforts consentis, aux progrès réalisés, les élèves comprennent les effets bénéfiques d'une activité physique régulière de plus en plus autonome tout au long de la vie. Par l'analyse réflexive sur les pratiques elles-mêmes et les conditions de la pratique, les élèves éviteront d'être des consommateurs naïs d'activités physiques et deviendront des pratiquants lucides et responsables, capables de réinvestir les effets de leur formation en dehors de l'école. A partir de la pratique physique et de la tenue des rôles sociaux (arbitre, juge, aide, etc.), l'EPS s'attache également à̀ faire construire les attitudes et comportements permettant de vivre en société : connaître les règles et en comprendre le sens, les respecter, les construire ensemble, pour agir en responsabilité.

Dans un contexte plus large, selon l'OCDE (2000), la littératie est l'aptitude à comprendre et à utiliser l'information écrite dans la vie courante, à la maison, au travail et dans la collectivité en vue 
d'atteindre des buts personnels et d'étendre ses connaissances et ses capacités. Chez les anglosaxons et au Québec, la littératie physique a pris une place importante en éducation physique et santé. Mais nous lui préférerons le concept de littératie motrice dont le sens nous semble plus extensif. La littératie motrice pourrait être l'aptitude à comprendre et à utiliser sa motricité et son corps dans la vie courante, les loisirs, les pratiques sportives et artistiques en vue d'atteindre des buts personnels et de rester physiquement actif. L'enjeu de la littératie motrice est bien celui de l'efficacité de nos actions en relation avec les connaissances disponibles.

Néanmoins, en mettant l'accent sur un déficit de compétences qui serait à l'origine de comportements peu favorables à la pratique physique, on risque d'oublier d'autres déterminants culturels, environnementaux ou sociaux. Dans les déterminants sociaux : le niveau d'éducation est le facteur corrélé le plus important mais il faut aussi considérer la situation socio-économique, le statut social, l'appartenance à un groupe minoritaire, l'âge, la dynamique du genre (Gréhaigne \& Caty, 2017 ; Gréhaigne \& Poggi, 2017). Cependant, la littératie motrice peut être envisagée à partir de quatre éléments clés :

- les connaissances qui entraînent la compréhension du contenu ;

- l'utilisation judicieuse des compétences motrices et de la pensée critique et créative ;

- une communication efficace pour transmettre des informations sous diverses formes ;

- une généralisation des connaissances et des compétences pour établir des mises en relation au sein et entre différents contextes.

Le but de l'apprentissage est, à côté des compétences motrices, la constitution de connaissances dans lesquelles la composante mentale, les représentations et les planifications de l'action motrice sont essentielles.

Nous allons maintenant aborder les différentes connaissances et compétences qui nous semblent indispensables pour mener à bien une vie physique d'adulte afin de continuer à jouer aux sports collectifs. Nous envisagerons successivement les compétences motrices puis les connaissances utiles à la perception et la gestion de l'affrontement. Ces connaissances et ces compétences doivent permettre à tous les adultes de réguler leur activité et de s'adapter à leurs possibilités du moment. En particulier, ils doivent pouvoir tenir compte de leur vieillissement dans le choix de leurs activités physiques. Une attitude critique est également nécessaire afin que les adultes puissent manifester un certain nombre d'attitudes, de dispositions, de connaissances afin d'évaluer leurs possibilités du moment.

\section{Compétences motrices et connaissances dans les sports collectifs}

Concernant l'enseignement des sports collectifs, les approches constructivistes permettent aux élèves d'acquérir des compétences pour se réaliser et, avec les autres, de vivre des expériences de 
motivation positive. Cette approche des jeux encourage les enseignants à respecter les différences individuelles ; encourager l'élaboration de compétences en matière de résolution de problèmes et de prise de décisions, encourager les élèves à construire leur propre sens à partir de leurs expériences en EPS et, enfin, encourager un réseau de compétences productif dans la formation des élèves (Gréhaigne \& Godbout, 1995).

Nous avons déjà développé les notions de compétences motrices et principes moteurs ainsi que les connaissances en sport collectif avec les règles d'action et de gestion de l'organisation du jeu (Gréhaigne \& Poggi, 2017). Il s'agit, ici, d'accroitre et mobiliser ses ressources pour enrichir sa motricité, la rendre efficace et favoriser la réussite. L'EPS doit conduire chaque élève à s'engager pleinement dans les apprentissages, quels que soient son niveau de pratique, sa condition physique et son degré d'inaptitude ou de handicap. Le développement des ressources, l'enrichissement de la motricité, la capacité à en disposer à bon escient dans le cadre d'une pratique raisonnée constituent des conditions nécessaires pour accroître la réussite de l'élève dans des contextes de pratiques diversifiées. Ils contribuent à l'équilibre personnel et à la réalisation de soi.

\section{Les compétences motrices}

Les élèves ou les joueurs possèdent un certain éventail de compétences motrices à leur disposition (celles de la vie courante ou d'autres beaucoup plus spécifiques et élaborées). Ces compétences motrices sont constituées d'habiletés qui peuvent être organisées autour de principes moteurs ayant trait à la capture, au transport et à la propulsion de la balle, c'est-à-dire un ensemble de manières d'agir, de gestes ou d'actions coordonnées qui présentent un caractère de rapidité et de stabilité. Nécessairement, ces compétences reposent sur un certain nombre de qualités physiques comme la force ou la vitesse, avec comme fondement théorique, les «principes moteurs » (Crouzillas \& Saladin, 2008). Ceux-ci sous-tendent le fonctionnement de ces compétences et sont organisés par :

- le caractère des tensions quand on veut doser et contrôler un mouvement explosif et moduler ses actions sur la balle ;

- la mise en tension / renvoi de la jambe libre quand on désire lancer loin le ballon (participation de plus en plus de segments à cette action, vers la construction de la chaîne motrice de la frappe de balle);

- le placement des chaînes musculaires pour optimiser les réponses motrices.

Il s'agit bien d'ajuster les compétences motrices construites par un joueur qui lui permettront, en jeu, l'échange, le transport et la propulsion de la balle. Les postures du joueur reposent sur un modèle dynamique basé sur des réflexes, des habitudes et des réponses adaptatives et fonctionnelles au jeu. Elles se traduisent par un processus actif qui maintient une disposition des différentes parties 
du corps dans l'espace. Dans ce cas, les postures et leurs décodages par les partenaires servent également de système de communication entre les joueurs.

A ce niveau, en continuant notre réflexion, nous pouvons parler de tactique individuelle plutôt que de technique car "à côté de la tactique collective, il y aussi une tactique individuelle, dénommée improprement par certains spécialistes "technique individuelle ». La technique sportive ne peut être qu'individuelle et elle a comme fondement la connaissance des procédés spécifiques de la manœuvre du ballon, ainsi que l'accomplissement de certains mouvements sans ballon» Teodorescu (1965/2013, p. 101). Pour compléter cette définition, nous dirons que la tactique individuelle, repose également sur les qualités cognitives du joueur ainsi que sur sa concentration et son aptitude à communiquer dans le jeu. Ainsi, la qualité de l'action tactique est aussi dépendante des connaissances et expériences des joueurs acquises à l'entraînement ou en compétition.

Pour les compétences motrices en sport collectif, un exemple d'un des éléments clé est constitué par la construction du lancer long relativement tendu ainsi que de son attraper en situation d'opposition. (Gréhaigne, Poggi, \& Zerai, 2017). En football, la circulation de la balle s'en trouve grandement facilitée avec un agrandissement de l'espace de jeu effectif occupé. De la sorte, un recours à la contre-attaque est plus aisé en permettant d'atteindre des joueurs placés loin à l'avant. Cela permet, également, de lire le jeu différemment, ce qui sera une aide à la constitution d'un répertoire de tactiques individuelles disponibles. Enfin, quand les conditions sont réunies, cela permet au joueur en possession du ballon de tirer au but avec une bonne chance de succès et non pas de faire une « passe » au gardien. L'augmentation du volume de manipulation dans toutes ses dimensions (haut, bas, antérieur, postérieur, latéral) détermine le volume d'utilisation de la balle qui, limité à la partie antérieure du buste et de la tête chez le débutant, va s'étendre progressivement dans toutes les dimensions de l'espace. La diversification des déplacements et la mobilité avec ou sans le ballon font que les déplacements souvent uniformes, orientés principalement selon l'axe longitudinal du terrain (couloir) en début d'apprentissage, vont se diversifier en direction et en distance (espace de jeu de plus en plus grand) et en intensité (changement de rythme). Une dissociation segmentaire et des ceintures permet d'atteindre une indépendance de plus en plus marquée favorisant une motricité monopodale. Il est à noter qu'en football, le pied tient lieu de main pour agir sur le ballon et le contrôler. Ici, la notion de «possession de la balle» doit être envisagée en tenant compte des échanges entre partenaires ou des conduites «balle au pied» par un joueur dans un espace ouvert. Enfin, il nous semble que cette approche qui part du concept de tactique individuelle ne dissocie pas tactique, technique et jeu. Ainsi, une construction de quelques objets de la technique (Gréhaigne, 2016) pourrait comprendre quelques notions simples :

- Différencier pousser et frapper un ballon. 
- Arrêter la balle : une des premières modalités visant à l'appropriation du ballon qui procède des mêmes problèmes moteurs que ci-dessus (poussée ou rétropoussée sur la balle).

- Frapper fort et / ou précis pour envoyer la balle à un partenaire (échange) ou tirer au but.

Tout cet ensemble est à mettre en relation avec la prise en compte des informations qui permettent la gestion et l'exécution de l'échange de balle au travers de la permutation de statut entre passeur et réceptionneur (Mérand, 1977; Gréhaigne, 2011). Nous allons en venir maintenant aux connaissances proprement dites.

\section{Les connaissances}

Au plan général, le terme connaissance désigne le contenu de la pensée qui correspond aux facultés mentales (perception, affectivité, intuition, pensée, concept, jugement, morale...) face à un objet envisagé et s'oppose à la foi ou l'illusion. Les connaissances se transforment, s'organisent, se combinent en fonction de chaque individu, de ses représentations de la réalité extérieure et du socle de connaissances qu'il possède. Concernant l'EPS, l'enseignement d'une forme scolaire des pratiques physiques, sportives et artistiques favorise l'accès à une culture raisonnée, critique et réfléchie des pratiques sociales et des valeurs qu'elle véhicule. L'EPS doit permettre aux élèves de disposer des connaissances nécessaires et d'un niveau de pratique suffisant pour aider à se situer au sein d'une culture contemporaine.

Pour les sports collectifs, pour identifier un jeu, on peut parler de règles constitutives, primaires ou de noyau central de règles qui définissent brièvement le but du jeu et qui interdisent que l'on puisse gagner en employant d'autres moyens que "jouer le jeu". Ainsi, les règles constitutives, puisqu'elles décrivent les moyens, fournissent au jeu ses caractères essentiels. Les règles constitutives aident à distinguer dans le jeu les exigences qu'il requiert et elles sont spécifiques pour chaque jeu. En plus des règles constitutives ou primaires, il existe des règles secondaires qui proviennent de l'expérience acquise en jeu et qui sont capables d'être changées sans altérer les caractères essentiels du jeu. Les règles secondaires peuvent faire l'objet d'interprétations différentes par les joueurs. La pensée tactique devient ici prépondérante car le gain du match nécessite la résolution de problèmes engendrés par l'affrontement (Malho, 1969 ; Deleplace, 1979).

De façon très pratique, les règles permettent aussi de mettre en relation les éléments du jeu pris en compte afin de résoudre les problèmes posés dans la pratique par une situation donnée en vue de répondre dans le jeu aux questions : Qui fait quoi ? Où ? Quand ? Comment ? Pourquoi ? Enfin, les règles d'action et les règles de gestion de l'organisation du jeu recouvrent un certain nombre de données qui ont trait, aussi, à la logique de l'activité et à des éléments d'organisation constitués au fil du développement de l'activité et de l'expérience des joueurs. 
En EPS, la nécessaire généralisation des apprentissages renvoie souvent à travailler plusieurs classes de problèmes. Une classe de problèmes est constituée par la similarité des données pratiques et des modes de résolutions auxquels l'élève doit faire appel, pour dénouer et relier les difficultés contenues dans plusieurs situations d'apprentissage. Celles-ci doivent avoir les caractéristiques suivantes : leurs éléments constitutifs ou l'organisation de ceux-ci sont semblables ou très voisins en nature, en nombre et en complexité et la classe de problèmes doit être accessible à l'élève et reconnue par lui comme telle. Ces situations devraient permettre de réorganiser les règles secondaires et de transformer les compétences motrices en vue d'une généralisation de ces connaissances car elles constituent un apport essentiel pour la construction de pratiques et de modes de résolution actualisés.

\section{Conclusion}

La littératie est généralement présentée comme un élément important de la culture et pour l'individu comme un moyen de communication efficace en relation avec sa capacité à comprendre le monde qui l'entoure. Dans le cas de la littératie motrice, la question clé reste: que signifie être physiquement éduqué ? Les jeux de balle sont une composante majeure des programmes actuels d'éducation physique dans le monde et peuvent être utilisés pour atteindre les objectifs actuels pour favoriser le développement comportemental, social et cognitif. La littératie motrice à propos des jeux en vue d'aider l'élève à « lire » le rapport des forces a été inspirée largement par la didactique des sports collectifs. Si un élève est bien formé, il ou elle dispose de connaissances qui lui permettent d'anticiper l'évolution des configurations du jeu. Il ou elle aura construit des compétences motrices et tactiques pour fournir des réponses appropriées aux rapports d'opposition. Enfin, il ou elle possèdera une expérience positive des jeux. Enfin, un examen systématique de la littérature mené par Edwards, Bryant, Keegan, Morgan et Jones (2016) a montré que le concept de littératie physique avait stimulé la recherche dans l'éducation physique, la participation sportive et la promotion de l'activité physique.

Aussi, l'objet de cet article était d'approfondir le concept de littératie motrice à propos de pratiques physiques sportives et artistiques et dans les jeux collectifs en particulier. Pour les formés, les règles d'action sont des connaissances sur les actions et, comme telles, peuvent être des outils de gestion des apprentissages. Elles constituent, avec les règles de gestion de l'organisation du jeu plus centrées sur l'analyse de la matière, un support à la culture tactique. Dans les sports d'équipe, les questions de tactique et d'intelligence collective sont fondamentales. Ainsi, l'élève doit construire des connaissances, des outils d'information et de décision pour jouer tout au long de sa vie, les connaissances acquises lui permettant de réguler son activité physique d'adulte. Avec l'âge, le résultat d'une rencontre ne dépend pas seulement de l'habileté des joueurs à agir sur une balle, mais 
surtout de leur adaptation et des choix tactiques effectués en relation avec la tactique individuelle de chacun.

Enfin, dans le cadre d'une littératie motrice, une autre question importante consiste à savoir comment les adultes ont appris. Ici, les chercheurs ont besoin d'approfondir ce que les joueurs apprennent effectivement et de décrire les mécanismes qui influencent les relations entre des connaissances, des compétences motrices et des performances sportives en constante progression.

\section{Bibliographie}

Almond, L. (2013). Physical literacy and fundamental movement skills: An introductory critique. Journal of Sport Science and Physical Education, 65, 81-89.

Crouzillas, Y. \& Saladin, P (2008). Apprentissage moteur et intelligence motrice. Questions à Alain Piron. Revue EP\&S, 329, 59-69.

Deleplace, R. (1979) Rugby de mouvement - Rugby total. Paris : Éducation Physique et Sports.

Edwards, L., Bryant, A., Keegan, R., Morgan, K., \& Jones, A. (2016). Definition, foundations and associa- tions of physical literacy: A systematic review. Sports Medicine, 1-14. doi:10.1007/s40279-016-0560-7

Gréhaigne, J.-F. (1996). Les règles d'action : un support pour les apprentissages. Éducation Physique et Sports, 260, 35-36.

Gréhaigne, J.-F. (Ed.). (2011). Des signes au sens. Le jeu, les indices, les postures et les apprentissages dans les sports collectifs à l'école. Besançon: Presses de l’Université de Franche-Comté.

Gréhaigne, J.F., \& Caty, D. (2017). Les élèves, la dynamique du genre et les sports collectifs. Revue EP\&S, 374, 27-29.

Gréhaigne, J.F., \& Guillon, R. (1991). Du bon usage des règles d'action. Échanges et controverses, 4, 43-66.

Gréhaigne, J-F., \& Poggi, M-P. (2017). Les connaissances et les compétences motrices en éducation physique : 1'exemple des sports collectifs. In J. Moniotte (Ed.) Réussir l'écrit 2 (pp. 191-204). Paris : Atlante

Gréhaigne, J-F., Poggi,M-P., \& Zerai, Z.(2017). Connaissances et compétences motrices en sport collectif : quoi enseigner ? eJRIEPS, 40, 143-162.

Groupe Sports Collectifs de L'Académie de Dijon (1994). Didactique des sports collectifs à l'école. Dossier Éducation Physique et Sport, 17.

Malho, F. (1969). L'acte tactique en jeu. Paris : Dunod. 
Mandigo, J. L., \& Holt, N. L. (2004). Reading the game. Introducing the notion of games literacy. Physical and Health Education Journal, 70 (3), 4-10.

Mérand, R. (1977). Considérations sur une problématique de rénovation des contenus de l'Education Physique en rapport avec les activités sportives contemporaines. In CPS de la FSGT (Ed.) L'éducateur face à la haute performance olympique (pp.05-27). Paris : Sport et Plein air.

Ministère de l'Education Nationale (2010). Programme lycée, 2010. Bulletin officiel spécial n 4 du 29 avril 2010.

OCDE (2000). La littératie à l'ère de l'information. Rapport final de l'enquête internationale sur la littératie des adultes.

Paillard, J. (1994). L'intégration sensori-motrice et idéo-motrice. In M. Richelle, J. Requin, \& M. Robert (Eds.), Traité de psychologie expérimentale (pp. 927-961). Paris : Presses Universitaires de France.

Samurçay, R., \& Pastre, P. (1995). La conceptualisation des situations de travail dans la formation des compétences. Éducation Permanente, 123, 13-31.

Teodorescu, L. (1965). Principes pour l'étude de la tactique commune aux jeux sportifs collectifs. Revue de la S.I.E.P.E.P.S., 3, 29-40. (texte publié à nouveau en 2013 dans la revue eJRIEPS, 28, 99-117).

Vergnaud, G. (2007). Définitions du concept de schème. Recherches en Education, 4, 17-22.

Zerai, Z. (2011). Apprentissage du handball chez les jeunes filles tunisiennes et françaises; apport de la verbalisation. Thèse (non publiée) en Science du Sport. Université de FrancheComté. 